\title{
Distributed Control for Alpha-Heterogeneous Dynamically Coupled Systems
}

\author{
Paolo Massioni \\ Laboratoire Ampère, UMR CNRS 5005, INSA de Lyon, Université de Lyon, 69621 Villeurbanne CEDEX, France
}

\begin{abstract}
This paper concerns the problem of distributed controller synthesis for a class of heterogeneous distributed systems composed of $\alpha$ ( 2 or more) different kinds of subsystems, interacting with one another according to a certain given graph topology. We will show that by employing Linear Matrix Inequalities (LMIs) tools, namely the full-block S-procedure, we can derive a control synthesis method based on $\mathcal{L}_{2}$ gain performance. This synthesis method guarantees stability and performance of a whole set of possible interconnection graphs, and its computational complexity does not depend on the number of subsystems involved but only on the number of different kinds of subsystems. The effectiveness of the new method is verified on a test case.
\end{abstract}

Keywords: Distributed Control, Heterogeneous Systems.

\section{Introduction}

The system and control community is devoting significant efforts on the development of distributed control methods for large scale systems, as it can be seen from the large number of works published in the field in the last 40 years [1, 3, 4, 6, 9, 10, 14]. By "distributed control", opposed to "centralized control", we mean a control action that is computed locally according to the physical spatial extension of the system, which is seen as an interconnection of simpler subsystems. The goal is replacing the high-order centralized controller with many simple (low-order) elementary controllers which only have access to a limited set of measures, for example only to those of the subsystems to which they are physically attached and their nearest neighbors.

This paper concerns the control of heterogeneous systems (see for example $[9,13,15]$ and references therein), made by the interconnection of $N$ subsystems (or agents), according to an interconnection structure described by a graph. We make the additional hypothesis of a certain regularity, namely we restrict to heterogeneous systems that are only made of a limited number $\alpha$ of different subsystem types, as shown in Fig. 1. For such systems, which we will call " $\alpha$-heterogeneous", we will consider the interconnection as an uncertainty (as done for example in [17]), and we will use a robust control tool (the full-block S-procedure [16]) to deal with it in the form of LMIs (Linear Matrix Inequalities). The contribution of this article is the fact that we show that such LMIs can be reduced to a set whose size does not depend on the number of subsystems $N$, but only on $\alpha$. This means that we obtain an analysis and synthesis method whose computational complexity is not depending on the number of subsystems, and which can virtually be applied even for

Email address: paolo.massioni@insa-lyon.fr (Paolo Massioni)

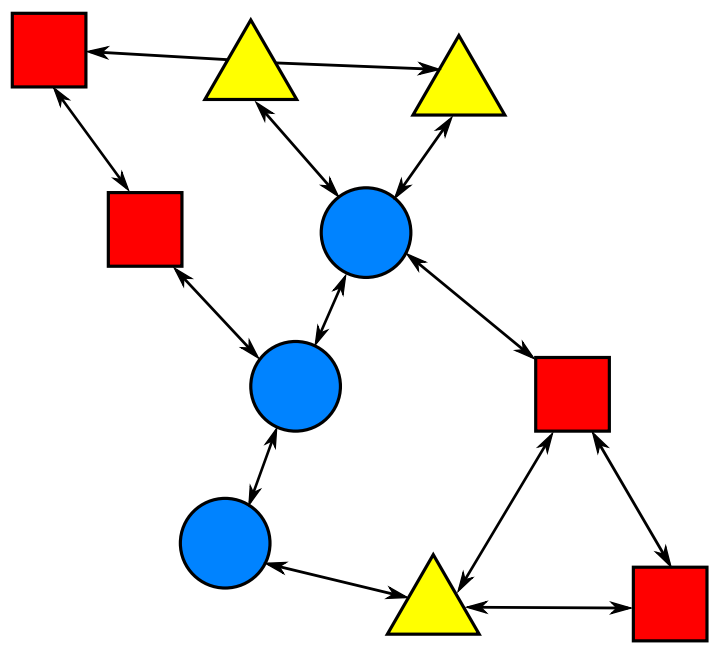

Figure 1: A heterogenous system made of the interconnection of subsystems of three different kinds. The arrows represent dynamic interactions among the subsystems.

$N \rightarrow \infty$, as long as the number of subsystem types $\alpha$ remains limited. This work ideally extends [11] and [12], which applied to homogeneous systems (the case of $\alpha=1$ ), and although we will focus on discrete-time systems, similar reasonings will work for continuous-time ones as well.

This article is organized as follows. Section 2 introduces the notation and the basic definitions, while Section 3 summarizes the full-block S-procedure. Section 4 contains the main result on the $\mathcal{L}_{2}$ gain analysis of $\alpha$-heterogeneous systems, and Section 5 shows how this result can be extended to distributed controller synthesis for such systems. Section 6 shows the application of the synthesis methods to an academic example, and then the conclusions are in Section 7. 


\section{Preliminaries}

\subsection{Notation}

Let $\mathbb{R}$ be the field of real numbers, $\mathbb{Z}$ the group of integer numbers, and $\mathbb{R}^{n \times m}$ the set of real $n \times m$ matrices. We denote the identity matrix of order $n$ by $I_{n}$ (or just $I$ if $n$ can be inferred from the context). The notation $A>0(A \prec 0)$ indicates that all the eigenvalues of the square matrix $A=A^{\top}$ are strictly positive (negative). Let $\bar{\sigma}(A)$ denote the maximum singular value of $A$.We will also use the symbol $*$ to denote entries that can be inferred from the symmetry of a matrix expression, and the symbol $\star$ in expressions of the type $X^{T} Q X$ to replace $X^{T}$ and avoid repetitions, i.e. $\star Q X=X^{T} Q X$. In this article we make extensive use of the Kronecker product [2], which we denote by the symbol $\otimes$; we remind one of its main properties, according to which:

$$
(A \otimes B)(C \otimes D)=(A C) \otimes(B D)
$$

if the dimensions of the matrices $A, B, C, D$ are compatible.

\section{2. $\alpha$-Heterogeneous systems}

We consider a class of systems which we call " $\alpha$ heterogeneous". Such systems are the result of the interconnection of $N=\sum_{i=1}^{\alpha} N_{i}$ subsystems of order $l$; these subsystems belong to $\alpha$ different classes, and $N_{i}$ elements are present for each class, according to the following definition.

Definition 1 ( $\alpha$-heterogeneous systems). Let $\mathcal{P}(k)$ be an $N \times N$ matrix, which we call the "pattern matrix", and which can be arbitrarily time-varying. We define $\theta_{j}=\sum_{i=1}^{j} N_{i}$ (with $\theta_{0}=0$ ) and $I_{\left\{a_{1}: a_{2}\right\}}$ as an $N \times N$ diagonal matrix which contains 1 in the the diagonal entries of indices from $a_{1}$ to $a_{2}$ (included) and 0 elsewhere. Let us consider an $\mathrm{Nl}$-th order linear discrete-time dynamical system of equations:

$$
\left\{\begin{array}{l}
x(k+1)=\mathcal{A} x(k)+\mathcal{B}_{w} w(k)+\mathcal{B}_{u} u(k) \\
z(k)=\mathcal{C}_{z} x(k)+\mathcal{D}_{z w} w(k)+\mathcal{D}_{z u} u(k) \\
y(k)=C_{y} x(k)+\mathcal{D}_{y w} w(k)
\end{array}\right.
$$

where $k \in \mathbb{Z}, x \in \mathbb{R}^{N l}$ is the state, $u \in \mathbb{R}^{N m_{u}}$ is the control input, $w \in \mathbb{R}^{N m_{w}}$ is the disturbance, $y \in \mathbb{R}^{N r_{y}}$ is the measured output and $z \in \mathbb{R}^{N r_{z}}$ is the performance output. We call such a system " $\alpha$-heterogeneous" (for a given $\alpha$ ) iff it has a state space realization with matrices of the kind:

$$
\mathcal{M}=\underbrace{\sum_{i=1}^{\alpha}\left(I_{\left\{\theta_{i-1}+1: \theta_{i}\right\}} \otimes M_{a}^{(i)}\right)}_{\overline{\mathcal{M}}}+\underbrace{\sum_{i=1}^{\alpha}\left(I_{\left\{\theta_{i-1}+1: \theta_{i}\right\}} \mathcal{P}(k) \otimes M_{b}^{(i)}\right)}_{\underline{\mathcal{M}}}
$$

where $\mathcal{M}$ represents any of the matrices in (2), and $\mathcal{P}(k)$ is the "pattern matrix"; the matrices $M_{a}^{(i)}$ are the diagonal blocks of $\mathcal{M}$, while the matrices $M_{b}^{(i)}$ constitute the off-diagonal blocks, according to the structure of $\mathcal{P}(k)$.

The matrices with superscript " $(i)$ " concern the dynamics of each of the $\alpha$ different kinds of systems. The elements of the state vector in entries from $1+(i-1) l$ to $i l$, with $1 \leqslant i \leqslant N$ can be considered as the state of the $i$-th subsystem, which belongs to type $\beta$ if $\theta_{\beta-1}+1 \leqslant i \leqslant \theta_{\beta}$. The block diagonal part of the matrices $(\overline{\mathcal{M}}$, made of the submatrices with the subscript " $a$ ") represents the internal dynamics of the subsystems, while the part depending on the pattern matrix $\mathcal{P}(k)(\mathcal{M}$, made of the submatrices with the subscript " $b$ ") accounts for the interactions between subsystems. A sparse pattern matrix indicates that each subsystem interacts only with a limited set of the others, e.g. its neighbors. There is no loss of generality in assuming that all the $\alpha$ different types are of the same order $l$, or have the same number of input/output channels, as one can add empty rows and columns to upgrade lower order systems to the higher one.

Alpha-heterogeneous systems can be written in a different equivalent form; such observation is reported in the form of a lemma.

Lemma 2. The system of equations:

$$
\left\{\begin{array}{l}
x(k+1)=\overline{\mathcal{A}} x(k)+\overline{\mathcal{B}}_{w} w(k)+\overline{\mathcal{B}}_{u} u(k)+\overline{\mathcal{B}}_{p} p(k) \\
z(k)=\overline{\mathcal{C}}_{z} x(k)+\overline{\mathcal{D}}_{z w} w(k)+\overline{\mathcal{D}}_{z u} u(k)+\overline{\mathcal{D}}_{z p} p(k) \\
y(k)=\overline{\mathcal{C}}_{y} x(k)+\overline{\mathcal{D}}_{y w} w(k)+\overline{\mathcal{D}}_{y p} p(k) \\
q(k)=\bar{C}_{q} x(k)+\overline{\mathcal{D}}_{q w} w(k)+\overline{\mathcal{D}}_{q u} u(k)
\end{array}\right.
$$

where all the matrices are block diagonal, and with $p, q \in$ $\mathbb{R}^{N m_{p}}$, and

$$
\begin{gathered}
\overline{\mathcal{B}}_{p}=\sum_{i=1}^{\alpha} I_{\left\{\theta_{i-1}+1: \theta_{i}\right\}} \otimes\left[\begin{array}{lll}
A_{b}^{(i)} & B_{w, b}^{(i)} & B_{u, b}^{(i)}
\end{array}\right] \\
\overline{\mathcal{D}}_{z p}=\sum_{i=1}^{\alpha} I_{\left\{\theta_{i-1}+1: \theta_{i}\right\}} \otimes\left[\begin{array}{lll}
C_{z, b}^{(i)} & D_{z w, b}^{(i)} & D_{z u, b}^{(i)}
\end{array}\right] \\
\overline{\mathcal{D}}_{y p}=\sum_{i=1}^{\alpha} I_{\left\{\theta_{i-1}+1: \theta_{i}\right\}} \otimes\left[\begin{array}{lll}
C_{y, b}^{(i)} & D_{y w, b}^{(i)} & 0
\end{array}\right] \\
\bar{C}_{q}=\sum_{i=1}^{\alpha} I_{\left\{\theta_{i-1}+1: \theta_{i}\right\}} \otimes\left[\begin{array}{lll}
I_{l} & 0 & 0
\end{array}\right]^{\top} \\
\overline{\mathcal{D}}_{q w}=\sum_{i=1}^{\alpha} I_{\left\{\theta_{i-1}+1: \theta_{i}\right\}} \otimes\left[\begin{array}{lll}
0 & I_{m_{w}} & 0
\end{array}\right]^{\top} \\
\overline{\mathcal{D}}_{q u}=\sum_{i=1}^{\alpha} I_{\left\{\theta_{i-1}+1: \theta_{i}\right\}} \otimes\left[\begin{array}{lll}
0 & 0 & I_{m_{u}}
\end{array}\right]^{\top},
\end{gathered}
$$

and $\overline{\mathcal{A}}, \overline{\mathcal{B}}_{w}, \overline{\mathcal{B}}_{u}, \bar{C}_{z}, \overline{\mathcal{D}}_{z w}, \overline{\mathcal{D}}_{z u}, \bar{C}_{y}, \overline{\mathcal{D}}_{y w}$ defined according to (3), is equivalent to (2) for

$$
p(k)=\left(\mathcal{P}(k) \otimes I_{m_{p}}\right) q(k) .
$$

Proof. Replace the expression of $p(k)$ in (6) into (4), and then simplify the resulting expression using the properties of the Kronecker product.

Remark 3. As all the matrices in (4) are block diagonal, the interconnections among the different subsystems are only in the relation (6) between $p(k)$ and $q(k)$. Notice also that from now on the symbols $A^{(i)}, B_{w}^{(i)}, B_{u}^{(i)}, B_{p}^{(i)}, C_{z}^{(i)}, C_{y}^{(i)}, C_{p}^{(i)}, D_{z w}^{(i)}, D_{z u}^{(i)}, D_{z p}^{(i)}$, $D_{y w}^{(i)}, D_{y p}^{(i)}$ will denote the diagonal blocks of the matrices in (4) corresponding to the $i$-th type of subsystem, which means the blocks between the $\left(\theta_{i-1}+1\right)$-th and the $\theta_{i}$-th (included). So, for example, $A^{(i)}=A_{a}^{(i)}, B_{p}^{(i)}=\left[\begin{array}{ccc}A_{b}^{(i)} & B_{w, b}^{(i)} & B_{u, b}^{(i)}\end{array}\right]$, and so on.

The system realization defined in (4), (5) and (6), with $m_{p}=$ $l+r_{y}+r_{u}$, is not necessarily minimal, in the sense that many of the entries of the signal $p(k)$ might be identical to zero; this means that the system can be reduced accordingly if any of the matrices defining the ones in (5) are always null. For example, 
if $B_{w, b}^{(i)}=0, D_{z w, b}^{(i)}=0$ and $D_{y w, b}^{(i)}=0$ for all $i$, then $\overline{\mathcal{D}}_{q w}=$ 0 and $\overline{\mathcal{B}}_{p}, \overline{\mathcal{D}}_{z p}$ and $\overline{\mathcal{D}}_{y p}$, etc. can be redefined accordingly by skipping the columns which would be identically zero. Notice that the system of Lemma 2 is in the typical Linear Fractional Transformation (LFT) form, with the interconnection part (the pattern matrix $\mathcal{P}(k))$ "pulled out" as an uncertainty.

\section{The Full Block S-Procedure}

In this paper we make use of a result from the so-called fullblock S-procedure. Before showing how we can exploit it, we briefly summarize the relevant $\mathcal{L}_{2}$ gain analysis result here.

Theorem 4 (Full Block S-Procedure [16]). Let us consider an uncertain discrete-time system described by the equations:

$$
\left\{\begin{array}{l}
x(k+1)=\mathrm{A} x(k)+\mathrm{B}_{\mathrm{p}} p(k)+\mathrm{B}_{\mathrm{w}} w(k) \\
q(k)=\mathrm{C}_{\mathrm{q}} x(k)+\mathrm{D}_{\mathrm{qp}} p(k)+\mathrm{D}_{\mathrm{qw}} w(t) \\
z(k)=\mathrm{C}_{\mathrm{z}} x(k)+\mathrm{D}_{\mathrm{zp}} p(k)+\mathrm{D}_{\mathrm{zw}} w(t)
\end{array}\right.
$$

where $x(k)$ is the state, $w(k)$ the disturbance, $z(k)$ the performance output, $q(k)$ and $p(k)$ signals for which it holds:

$$
p(k)=\Delta q(k)
$$

where $\Delta$ is an arbitrarily fast time-varying uncertainty which assumes values in the convex hull generated by a set $\left\{\Delta_{j}\right\}$ for $j=0, \ldots, J$, with $\Delta_{0}=0$. The systems has an $\mathcal{L}_{2}$ gain from $w$ to $z$ smaller than $\gamma$ for all the valid uncertainties if the following LMI set is feasible

$$
\mathrm{Y}>0, \mathrm{R}>0,
$$

$$
\star\left[\begin{array}{cc}
\mathrm{Q} & \mathrm{S} \\
\mathrm{S}^{\top} & \mathrm{R}
\end{array}\right]\left[\begin{array}{c}
I \\
-\Delta_{j}^{\top}
\end{array}\right] \prec 0 \text { for } j=0, \ldots, J
$$

$$
\star\left[\begin{array}{cc|cc|cc}
-\mathrm{Y} & 0 & 0 & 0 & 0 & 0 \\
0 & \mathrm{Y} & 0 & 0 & 0 & 0 \\
\hline 0 & 0 & \mathrm{Q} & \mathrm{S} & 0 & 0 \\
0 & 0 & \mathrm{~S}^{\top} & \mathrm{R} & 0 & 0 \\
\hline 0 & 0 & 0 & 0 & -I & 0 \\
0 & 0 & 0 & 0 & 0 & \gamma^{2} I
\end{array}\right]\left[\begin{array}{ccc}
-\mathrm{A}^{\top} & -\mathrm{C}_{\mathrm{q}}^{\top} & -\mathrm{C}_{\mathrm{z}}^{\top} \\
I & 0 & 0 \\
-\mathrm{B}_{\mathrm{p}}^{\top} & -\mathrm{D}_{\mathrm{qp}}^{\top} & -\mathrm{D}_{\mathrm{zp}}{ }^{\top} \\
0 & I & 0 \\
-\mathrm{B}_{\mathrm{w}}^{\top} & -\mathrm{D}_{\mathrm{qw}}^{\top} & -\mathrm{D}_{\mathrm{zw}}{ }^{\top} \\
0 & 0 & I
\end{array}\right]>0
$$

where $\mathrm{Y}=\mathrm{Y}^{\top}, \mathrm{R}=\mathrm{R}^{\top}, \mathrm{S}, \mathrm{Q}=\mathrm{Q}^{\top}$ are the decision variables.

If we look at this last theorem and compare it to an $\alpha$ heterogeneous system in the form of Lemma 2, then it is apparent that the full-block S-procedure can be used directly for the $\mathcal{L}_{2}$ gain analysis and eventually synthesis. Such direct use of the S-procedure would yield LMIs whose size is depending on the number of subsystems $N$, as one would need to plug in the matrices of (4) into (10) and (11), with $\left\{\Delta_{j}\right\}=\left\{\mathcal{P}(k) \otimes I_{m_{p}}\right\}$, according to (6). In the next section, we will show how the addition of a few constraints will allow a significant reduction of the LMIs' size.

\section{4. $\mathcal{L}_{2}$ gain analysis for $\alpha$-heterogeneous systems}

\subsection{Main result}

Our main result concerns the $\mathcal{L}_{2}$ gain performance analysis of $\alpha$-heterogeneous systems.

Theorem 5. Consider an $\alpha$-heterogeneous system in the form of Lemma 2. The system has an $\mathcal{L}_{2}$ gain from $w$ to $z$ smaller than $\gamma$ if the following LMI set is feasible

$$
R>0
$$

$$
\star\left[\begin{array}{cc}
Q & 0 \\
0 & R
\end{array}\right]\left[\begin{array}{c}
I_{m_{p}} \\
\bar{\delta} I_{m_{p}}
\end{array}\right] \prec 0, \quad Q \prec 0
$$

$$
\left[\begin{array}{cccccc}
Y^{(i)} & 0 & 0 & -Y^{(i)} A^{(i) \top} & -Y^{(i)} C_{q}^{(i) \top} & -Y^{(i)} C_{z}^{(i) \top} \\
* & -Q & 0 & -Q B_{p}^{(i) \top} & 0 & -Q D_{z p}^{(i) \top} \\
* & * & I_{m_{w}} & -B_{w}^{(i) \top} & -D_{q w}^{(i) \top} & -D_{z w}^{(i) \top} \\
* & * & * & Y^{(i)} & 0 & 0 \\
* & * & * & * & R & 0 \\
* & * & * & * & * & \gamma^{2} I_{r_{z}}
\end{array}\right]>0
$$

where $Y^{(i)}=Y^{(i) \top} \in \mathbb{R}^{l \times l}$, and $R=R^{\top}, Q=Q^{\top} \in \mathbb{R}^{m_{p} \times m_{p}}$ are the decision variables (and the other matrices have been defined in Remark 3$)$, and $\bar{\delta} \geqslant \bar{\sigma}(\mathcal{P}(k))$, for all valid $k \in \mathbb{Z}$.

Proof. As noted earlier on, it is possible to directly use the fullblock S-procedure (Theorem 4) on the system in the form of Lemma 2. Let us start analyzing the first of the two sets of LMIs involved, namely (10). According to (6), we have that $\left\{\Delta_{j}\right\}=\left\{\mathcal{P}(k) \otimes I_{m_{p}}\right\} \cup\{0\}$. We then restrict the multipliers to be of the form $\mathrm{Q}=I_{N} \otimes Q, \mathrm{R}=I_{N} \otimes R$ (as done in [8]), with $Q=Q^{\top}, R=R^{\top}$; this step involves conservatism. We also assume $S=0$, but this step does not necessarily involve conservatism, as it will be explained later on at the end of the proof. At this point, the size of the LMI in (10) is proportional to the number of subsystems $N$. We then show that under the hypothesis of the chosen form of the multiplier, (13) $\Rightarrow$ (10).

The case of $\Delta_{0}=0$ immediately yields $Q \prec 0$. For the remaining cases of $\Delta_{j}=\mathcal{P}(k) \otimes I_{m_{p}}$, from (10) by working out the matrix product we get

$$
I_{N} \otimes Q+\mathcal{P}(k) \mathcal{P}(k)^{\top} \otimes R<0
$$

We use a singolar value decomposition (SVD) of the matrix $\mathcal{P}(k)$, for which $\mathcal{P}(k)=\mathcal{U}(k) \Sigma(k) \mathcal{V}(k)^{\top}$, where $\Sigma(k) \in \mathbb{R}^{N \times N}$ is a diagonal matrix (the matrix of the singular values), and $\mathcal{U}(k) \mathcal{U}(k)^{\top}=\mathcal{U}(k)^{\top} \mathcal{U}(k)=\mathcal{V}(k) \mathcal{V}(k)^{\top}=\mathcal{V}(k)^{\top} \mathcal{V}(k)=I_{N}$. Replacing the SVD expression of $\mathcal{P}(k)$ into (15), we get

$$
I_{N} \otimes Q+\mathcal{U}(k) \Sigma(k)^{2} \mathcal{U}(k)^{\top} \otimes R<0
$$

which becomes

$$
I_{N} \otimes Q+\Sigma(k)^{2} \otimes R<0
$$


through a congruent transformation of matrix $\mathcal{U}(k) \otimes I_{m_{p}}$. At last, by a Schur complement argument, (17) is equivalent to

$$
\left[\begin{array}{cc}
I_{N} \otimes Q & \Sigma(k) \otimes R \\
* & -I_{N} \otimes R
\end{array}\right] \prec 0 .
$$

This last inequality contains only block-diagonal terms, as $\Sigma(k)$ and $I_{N}$ are block-diagonal. This implies that (18) is equivalent to the following set of LMIs

$$
\left[\begin{array}{cc}
Q & \sigma_{v}(k) R \\
* & -R
\end{array}\right]<0,
$$

where the $\sigma_{v}(k)$, for $v=1, \ldots, N$, are the singular values of $\mathcal{P}(k)$. The expression is affine in $\sigma_{v}(k)$, so if the expression holds for the extreme values of $\sigma_{v}(k)$, then it will hold for all the intermediate values. As we have that $0 \leqslant \sigma_{\nu}(k) \leqslant \bar{\delta}$, this means that

$$
\left[\begin{array}{cc}
Q & \bar{\delta} R \\
* & -R
\end{array}\right] \prec 0, \quad Q \prec 0,
$$

implies (19) for all possible values of $\sigma_{v}(k)$. The equations in (20) are equivalent to those in (13) by a Schur argument. So far we have proven that (13) $\Leftrightarrow(20) \Rightarrow$ (19) $\Leftrightarrow$ (18) $\Rightarrow$ (10).

At last, we remark that the matrices in (11) are all block diagonal with repeated blocks, so (11) is immediately equivalent to a set of only $\alpha$ different LMIs; these LMIs can be put into the form of (14) by developing the product and then applying a Schur complement.

This analysis result allows finding an upper bound of the $\mathcal{L}_{2}$ gain of the global system, whose order is proportional to $N$, by solving a set of LMIs whose size does not depend on $N$ but only on the number of different types of subsystems $\alpha$ and the maximum order and number of input/output channel of each of them. Moreover, notice that $\mathcal{P}(k)$ is not present in the LMIs: the geometry of the interconnection boils down to the sole parameter $\bar{\delta}$. Then the upper bound on the $\mathcal{L}_{2}$ gain will be valid for all system interconnection topologies whose pattern matrix $\mathcal{P}(k)$ has the singular values bounded by $\bar{\delta}$.

Remark 6. In the proof of Theorem 5, we have assumed that the term $\mathrm{S}$ is equal to 0 . This assumption might look like a source of additional conservatism, but actually it is not, for the case of our result. In fact, Theorem 5 focuses only on the size of the uncertainty $\Delta_{j}$, in our case $\mathcal{P}(k) \otimes I_{m_{p}}$, as it is described by the maximum singular value. So it means that if we account for a given uncertainty $\bar{\Delta}$, we will account also for its negative value $-\bar{\Delta}$ (i.e., if we allow a certain $\mathcal{P}(k)$, we allow $-\mathcal{P}(k)$ as well). This means that (10) will yield both:

$$
\mathrm{Q}-\mathrm{S} \bar{\Delta}^{\top}-\bar{\Delta} \mathrm{S}^{\top}+\bar{\Delta} \mathrm{R} \bar{\Delta}^{\top}
$$

and

$$
\mathrm{Q}+\mathrm{S} \bar{\Delta}^{\top}+\bar{\Delta} \mathrm{S}^{\top}+\bar{\Delta} \mathrm{R} \bar{\Delta}^{\top}
$$

These two inequalities turn into each other if we replace $\mathrm{S}$ with -S. The same holds for (11), we get the same inequality if we replace $\mathrm{S}$ with $-\mathrm{S}$, if we consider that, due to the symmetry of the problem, we can replace $p(k)$ with $-p(k)$, i.e. $\mathrm{B}_{\mathrm{p}}$ with $-\mathrm{B}_{\mathrm{p}}, \mathrm{D}_{\mathrm{qp}}$ with $-\mathrm{D}_{\mathrm{qp}}$, and $\mathrm{D}_{\mathrm{zp}}$ with $-\mathrm{D}_{\mathrm{zp}}$. Considering that the optimal solution to the LMI problem is unique, this means that any result for which $\mathrm{S} \neq-\mathrm{S}$ would lead to a contradiction. So the optimal solution has $\mathrm{S}=-\mathrm{S}=0$.

\section{5. $\mathcal{L}_{2}$ gain synthesis for $\alpha$-heterogeneous systems}

From Theorem 5 the step to synthesis results is rather immediate. We then report the case of state feedback as well as the more involved output feedback.

\subsection{State feedback}

We consider a first feedback law of the kind

$$
u(k)=\sum_{i=1}^{\alpha}\left(I_{\left\{\theta_{i-1}+1: \theta_{i}\right\}} \otimes K^{(i)}\right) x(k),
$$

which coincides with local feedback loops of gain $K^{(i)}$ for each system of type $i$. The local closed-loop system matrices become

$$
\begin{array}{cl}
A_{c l}^{(i)}=A^{(i)}+B_{u}^{(i)} K^{(i)}, & B_{w, c l}^{(i)}=B_{w}^{(i)}, \\
C_{z, c l}^{(i)}=C_{z}^{(i)}+D_{z u}^{(i)} K^{(i)}, & D_{z w, c l}^{(i)}=D_{z w}^{(i)},
\end{array}
$$

(the others are unchanged); replacing them into the expressions (12), (13) and (14) yields immediately the state-feedback synthesis method, which we summarize in the following theorem.

Theorem 7. Consider an $\alpha$-heterogeneous system in the form of Lemma 2. There exists a control law as in (23) for which the system has a closed-loop $\mathcal{L}_{2}$ gain from $w$ to $z$ smaller than $\gamma$ if the LMI set in (25) and (26) (at the top of the next page) is feasible, where $Y^{(i)}=Y^{(i) \top} \in \mathbb{R}^{l \times l}, R=R^{\top}, Q=Q^{\top} \in \mathbb{R}^{m_{p} \times m_{p}}$ and $L^{(i)} \in \mathbb{R}^{m_{u} \times l}$ are the decision variables, and $\bar{\delta} \geqslant \bar{\sigma}(\mathcal{P}(k))$, for all valid $k \in \mathbb{Z}$. The controller gains can be obtained from the relation $K^{(i)}=L^{(i)} Y^{(i)-1}$.

Proof. Replace the matrices of (24) into (14), then set $L^{(i)}=$ $K^{(i)} Y^{(i)}$.

\subsection{Outputfeedback}

In the output-feedback case, the controller is of equations

$$
\left\{\begin{aligned}
x_{c}(k)= & \sum_{i=1}^{\alpha}\left(I_{\left\{\theta_{i-1}+1: \theta_{i}\right\}} \otimes A_{c}^{(i)}\right) x_{c}(k)+ \\
& +\sum_{i=1}^{\alpha}\left(I_{\left\{\theta_{i-1}+1: \theta_{i}\right\}} \otimes B_{c}^{(i)}\right) y(k) \\
u(k)= & \sum_{i=1}^{\alpha}\left(I_{\left\{\theta_{i-1}+1: \theta_{i}\right\}} \otimes C_{c}^{(i)}\right) x_{c}(k)+ \\
& +\sum_{i=1}^{\alpha}\left(I_{\left\{\theta_{i-1}+1: \theta_{i}\right\}} \otimes D_{c}^{(i)}\right) y(k) .
\end{aligned}\right.
$$

In this case, to our knowledge, the synthesis cannot be solved by LMIs. It will be necessary to solve Bilinear Matrix Inequalities (BMIs) instead, as summarized in the following last theorem. 


$$
\begin{gathered}
R>0, \quad \star\left[\begin{array}{cc}
Q & 0 \\
0 & R
\end{array}\right]\left[\begin{array}{c}
I_{m_{p}} \\
\bar{\delta} I_{m_{p}}
\end{array}\right] \prec 0, \quad Q<0, \\
{\left[\begin{array}{cccccc}
Y^{(i)} & 0 & 0 & -Y^{(i)} A^{(i) \top}-L^{(i) \top} B_{u}^{(i) \top} & -Y^{(i)} C_{q}^{(i) \top}-L^{(i) \top} D_{q u}^{(i) \top} & -Y^{(i)} C_{z}^{(i) \top}-L^{(i) \top} D_{z u}^{(i) \top} \\
* & -Q & 0 & -Q B_{p}^{(i) \top} & 0 & -Q D_{z p}^{(i) \top} \\
* & * & I_{m_{w}} & -B_{w}^{(i) \top} & -D_{q w}^{(i) \top} & -D_{z w}^{(i) \top} \\
* & * & * & Y^{(i)} & 0 & 0 \\
* & * & * & * & R & 0 \\
* & * & * & * & \text { for } i=1, \ldots, \alpha & \gamma^{2} I_{r_{z}}
\end{array}\right]>0}
\end{gathered}
$$

Theorem 8. Consider an $\alpha$-heterogeneous system in the form of Lemma 2. There exists a controller as in (27) for which the system has a closed-loop $\mathcal{L}_{2}$ gain from $w$ to $z$ smaller than $\gamma$ if the set of BMIs in (29) and (30) (at the top of the next page) is feasible, where $X^{(i)}=X^{(i) \top}, Z^{(i)}=Z^{(i) \top}, H^{(i)} \in \mathbb{R}^{l \times l}, L^{(i)} \in$ $\mathbb{R}^{m_{u} \times l}, J^{(i)} \in \mathbb{R}^{m_{u} \times r_{y}}, F^{(i)} \in \mathbb{R}^{l \times r_{y}}$, and $R=R^{\top}, Q=Q^{\top} \in$ $\mathbb{R}^{m_{p} \times m_{p}}$ are the decision variables, $\bar{\delta} \geqslant \bar{\sigma}\left(\mathcal{P}_{k}\right)$, for all valid $k \in \mathbb{Z}$. The controller state-space matrices as in (27) can be obtained from the following relations:

$$
\begin{aligned}
D_{c}^{(i)}= & J^{(i)} \\
C_{c}^{(i)}= & \left(L^{(i)}-J^{(i)} C_{y}^{(i)} X^{(i)}\right) U^{(i)-1} \\
B_{c}^{(i)}= & V^{(i)-1}\left(F^{(i)}-Z^{(i)} B_{u}^{(i)} J^{(i)}\right) \\
A_{c}^{(i)}= & V^{(i)-1}\left(H^{(i)}-Z^{(i)}\left(A^{(i)}+B_{u}^{(i)} J^{(i)} C_{y}^{(i)}\right) X^{(i)}-\right. \\
& \left.-V^{(i)} B_{c}^{(i)} C_{y}^{(i)} X^{(i)}\right) U^{(i)-1}-V^{(i)-1} Z^{(i)} B_{u}^{(i)} C_{c}^{(i)},
\end{aligned}
$$

where $V^{(i)}, U^{(i)}$ are nonsingular matrices for which $V^{(i)} U^{(i)}=$ $I_{l}-Z^{(i)} X^{(i)}$.

Proof. The BMI in (30) derives from (14) through the application of the standard controller-linearizing transformation, which can be found in [7] or [5].

This last theorem is less readily applicable than Theorem 7 as it involves BMIs, for which the convex optimizations solvers are not applicable. One possibility for solving such BMIs is to iteratively solve a set of LMIs deriving from them where alternatively a part of the decision variables is kept constant [16]. The following algorithm is an example of such a strategy.

Algorithm 9. Choose an integer $g>0$. Set $\rho:=1 / g$.

1. Find the nominal controller by minimizing $\gamma$ under the constraints in (30) to which the third and the seventh block rows and columns have been removed; store $\bar{X}^{(i)}:=X^{(i)}$, $\bar{Z}^{(i)}:=Z^{(i)}, \bar{H}^{(i)}:=H^{(i)}, \bar{L}^{(i)}:=L^{(i)}, \bar{J}^{(i)}:=J^{(i)}, \bar{F}^{(i)}:=F^{(i)}$.

2. Minimize $\gamma$ under (30) and

$$
\star\left[\begin{array}{cc}
Q & 0 \\
0 & R
\end{array}\right]\left[\begin{array}{c}
I_{m_{p}} \\
\rho \bar{\delta} I_{m_{p}}
\end{array}\right] \prec 0, \quad Q \prec 0, \quad R>0
$$

with the additional constraint of $X^{(i)}=\bar{X}^{(i)}, Z^{(i)}=\bar{Z}^{(i)}$, $H^{(i)}=\bar{H}^{(i)}, L^{(i)}=\bar{L}^{(i)}, J^{(i)}=\bar{J}^{(i)}, F^{(i)}=\bar{F}^{(i)}$; store $\bar{Q}:=Q$, $\bar{R}:=R$.
3. Minimize $\gamma$ under (30), under the additional constraint of $Q=\bar{Q}, R=\bar{R}$; store $\bar{X}^{(i)}:=X^{(i)}, \bar{Z}^{(i)}:=Z^{(i)}, \bar{H}^{(i)}:=H^{(i)}$, $\bar{L}^{(i)}:=L^{(i)}, \bar{J}^{(i)}:=J^{(i)}, \bar{F}^{(i)}:=F^{(i)}$.

4. If $\rho=1$ terminate, otherwise $\rho:=\rho+1 / g$ (increment $\rho$ of $1 / g$ ) and go to 2 .

If at any time during the algorithm an LMI problem is found to be unfeasible, terminate (the desired controller does not exist or it cannot be found).

The working principle of the algorithm is basically to start by finding the nominal controller (no uncertainty, or $\bar{\delta}=0$ ), and then expand gradually the size of the uncertainty until it reaches the desired one. The procedure is not guaranteed to arrive at a solution but it has been verified to work in practice, at least in our test case as shown in the following section.

\section{Simulation Results}

In order to show the effectiveness of the method, we test in simulation both the state-feedback and the output-feedback synthesis methods. We consider a 2-heterogeneous system made of two very different kinds of subsystems, for which the internal dynamics is described by the matrices

$$
A_{a}^{(1)}=\left[\begin{array}{cc}
1 & 0 \\
1 & 0.95
\end{array}\right], A_{a}^{(2)}=\left[\begin{array}{cc}
0.6 & 0.8 \\
-0.8 & 0.6
\end{array}\right] .
$$

Notice that type 1 contains an integrator, whereas type 2 is an undamped oscillator. We do not report the other matrices of the system due to space constraints. We choose $N_{1}=N_{2}=5$ and we consider a random time-invariant sparse pattern matrix $\mathcal{P}$ with $\bar{\sigma}(\mathscr{P}) \leqslant \bar{\delta}=3$, for which the global, uncontrolled systems is unstable.

We have computed the distributed controller of this article and we have compared its performance with a standard centralized $\mathcal{L}_{2}$-gain optimal controller. The results are in Table 1 .

We can see that the state-feedback case is very conservative, leading to a performance that is roughly three times worse than the centralized controller. This can be explained also by the fact that the distributed state-feedback controller is actually a decentralized controller (it does not use information on the neighbors in order to control an element), as it is apparent by looking at (23). The output-feedback controller, which is truly distributed, 


$$
\begin{aligned}
& R>0, \quad \star\left[\begin{array}{cc}
Q & 0 \\
0 & R
\end{array}\right]\left[\begin{array}{c}
I_{m_{p}} \\
-\bar{\delta} I_{m_{p}}
\end{array}\right] \prec 0, \quad Q \prec 0, \\
& {\left[\begin{array}{cccccccc}
X^{(i)} & I_{l} & 0 & 0 & -X^{(i)} A^{(i) \top}-L^{(i) \top} B_{u}^{(i) \top} & -H^{\top} & -X^{(i)} C_{q}^{(i) \top}-L^{(i) \top} D_{q u}^{(i) \top} & -X^{(i)} C_{z}^{(i) \top}-L^{(i) \top} D_{z u}^{(i) \top} \\
* & Z^{(i)} & 0 & 0 & -A^{(i) \top}-C_{y}^{(i) \top} J^{(i) \top} B_{u}^{(i) \top} & -A^{(i) \top} Z^{(i)}-C_{y}^{(i) \top} F^{(i) \top} & -C_{q}^{(i) \top}-C_{y}^{(i) \top} J^{(i) \top \top} D_{q u}^{(i) \top} & -C_{z}^{(i) \top}-C_{y}^{(i) \top} J^{(i) \top} D_{z u}^{(i) \top} \\
* & * & -Q & 0 & -Q B_{p}^{(i) \top}-Q D_{y p}^{(i) \top} J^{(i) \top} B_{u}^{(i) \top} & -Q B_{p}^{(i) \top} Z^{(i)}-Q D_{y p}^{(i) \top} F^{(i) \top} & 0 & -Q D_{z p}^{(i) \top}-Q D_{y p}^{(i) \top} J^{(i) \top} D_{z u}^{(i) \top} \\
* & * & * & I_{m_{w}} & -B_{w}^{(i) \top}-D_{y w}^{(i) \top} J^{(i) \top} B_{u}^{(i) \top} & -B_{w}^{(i) \top} Z^{(i)}-D_{y w}^{(i) \top} F^{(i) \top} & -D_{q w}^{(i) \top}-D_{y w}^{(i) \top} J^{(i) \top} D_{q u}^{(i) \top} & -D_{z w}^{(i) \top}-D_{y w}^{(i) \top} J^{(i) \top} D_{z u}^{(i) \top} \\
* & * & * & * & X^{(i)} & I_{l} & 0 & 0 \\
* & * & * & * & * & Z^{(i)} & 0 & 0 \\
* & * & * & * & * & * & R & -0 \\
* & * & * & * & * & * & * & \gamma^{2} I_{r_{z}}
\end{array}\right]>0}
\end{aligned}
$$

for $i=1 \ldots \alpha$

\begin{tabular}{|c|c|c|}
\hline & $\begin{array}{c}\text { state } \\
\text { feedback }\end{array}$ & $\begin{array}{c}\text { output } \\
\text { feedback }\end{array}$ \\
\hline$\frac{\mathcal{L}_{2} \text { gain of distributed controller }}{\mathcal{L}_{2} \text { gain of centralized controller }}$ & 2.92 & 1.06 \\
\hline
\end{tabular}

Table 1: Simulation results: performance of the distributed controller compared to the centralized one.

has instead a performance which is only $6 \%$ worse than the centralized counterpart, reassuring us that the conservatism is rather limited.

We have considered a time-invariant $\mathcal{P}$ in order to be able to compute and compare the norms; nevertheless, we have verified that the distributed controller works under arbitrarily timevarying pattern matrices satisfying $\bar{\sigma}(\mathcal{P}(k)) \leqslant 3$, with the same disturbance attenuation properties.

\section{Conclusion}

We have introduced a new method for controlling a class of distributed (or multi-agent) systems made of the interconnection of different kinds of subsystems. The method is based on the full-block S-procedure and its interesting aspect is the fact that its computational complexity does not depend on the size of the global system, but only on the number of types of subsystems involved. This light computational cost comes at the cost of some conservatism, which is apparent in the proof of Theorem 5, as the multipliers for the S-procedure are constrained to a special form. Nevertheless, it has been verified on a simulation that the performance loss with respect to a centralized controller is small, thus not compromising the usability of the result.

\section{References}

[1] B. Bamieh, F. Paganini, and M.A. Dahleh. Distributed control of spatially invariant systems. IEEE Trans. Aut. Control, 47(7):1091-1107, 2002.

[2] J.W. Brewer. Kronecker products and matrix calculus in system theory. IEEE Trans. Circuits Syst., 25(9), September 1978.
[3] R. D'Andrea and G.E. Dullerud. Distributed control design for spatially interconnected systems. IEEE Trans. Aut. Control, 48(9):1478-1495, September 2003.

[4] E.J. Davison and W. Gesing. Sequential stability and optimization of large scale decentralized systems. Automatica, 15(3):307-324, 1979.

[5] M.C. de Oliveira, J.C. Geromel, and J. Bernoussou. An LMI optimization approach to multiobjective controller design for discrete-time systems. In Proc. of the 38th Conference on Decision E Control, Phoenix, Arizona, USA, December 1999.

[6] J.A. Fax and R.M. Murray. Information flow and cooperative control of vehicle formations. IEEE Trans. Aut. Control, 49(9), 2004.

[7] P. Gahinet. Explicit controller formulas for LMI-based $\mathcal{H}_{\infty}$ synthesis. Automatica, 32(7):1007-1014, 1996.

[8] C. Hoffmann, A. Eichler, and H. Werner. Distributed control of linear parameter-varying decomposable systems. In American Control Conference (ACC), 2013, pages 2380-2385, 2013.

[9] C. Langbort, R.S. Chandra, and R. D'Andrea. Distributed control design for systems interconnected over an arbitrary graph. IEEE Trans. Aut. Control, 49(9):1502-1519, September 2004.

[10] Z. Li, Z. Duan, G. Chen, and L. Huang. Consensus of multiagent systems and synchronization of complex networks: A unified viewpoint. IEEE Trans. Circuits Syst. Regul. Pap., 57(1):213-224, 2010.

[11] P. Massioni and M. Verhaegen. Distributed control for identical dynamically coupled systems: a decomposition approach. IEEE Trans. Aut. Control, 54(1):124-135, January 2009.

[12] P. Massioni and M. Verhaegen. A full block S-procedure application to distributed control. In Proc. of the 2010 American Control Conference, pages 2338-2343, Baltimore, USA, 2010.

[13] N. Motee and A. Jadbabaie. Optimal control of spatially distributed systems. IEEE Trans. Aut. Control, 53(7):1616-1629, August 2008.

[14] A.P. Popov and H. Werner. A robust control approach to formation control. In Proc. of the 10th European Control Conference, Budapest, Hungary, August 2009.

[15] J.K. Rice and M. Verhaegen. Distributed control: a sequentially semiseparable approach for spatially heterogeneous linear systems. IEEE Trans. Aut. Control, 54(6):1270-1283, 2009.

[16] C.W. Scherer. LPV control and full block multipliers. Automatica, 37(3):361-375, March 2001.

[17] G. Scorletti and G. Duc. An LMI approach to decentralized $\mathcal{H}_{\infty}$ control. Int. J. Control, 74(3):211-224, 2001. 